\title{
Oportunidades de aprendizaje y formación docente: una mirada desde las Ecologías de Aprendizaje
}

\section{Opportunities for teacher training: an approach from the Learning Ecologies}

\author{
Alba Souto-Seljo*1 \\ alba.souto@unir.net \\ IRIS ESTÉVEZ** \\ iris.estevezb@udc.es \\ Olalla Sande** \\ olalla.sande.blanco@udc.es \\ * Universidad Internacional de La Rioja, España \\ **Universidade da Coruña, España
}

\section{Resumen:}

Una de las características más relevantes de la Sociedad de la Información y del Conocimiento es que el aprendizaje ya no solo tiene lugar en las instituciones formativas regladas, sino que también se halla en espacios no formales e informales. Las tecnologías digitales nos brindan la posibilidad de aprender en cualquier momento y en cualquier lugar, rompiendo las barreras espacio-temporales por lo que, actualmente, las experiencias de aprendizaje son ilimitadas. Así pues, el presente trabajo tiene como objetivo analizar cómo las diferentes oportunidades de aprendizaje y formación contribuyen al desarrollo profesional de los docentes. Esta investigación, de corte cualitativo, se ha desarrollado a través de la tradición de investigación de Estudio de Caso. Los participantes que conforman el caso son cuatro docentes

\begin{abstract}
:
One of the most relevant characteristics of the Information and Knowledge Society is the fact that learning no longer takes place merely in regulated training institutions, but also in non-formal and informal spaces. Digital technologies give us the possibility to learn at any time and in any place. This breaks space-time barriers generating learning experiences which nowadays are unlimited. Thus, the present work aims to analyse how the different learning and training opportunities contribute to the professional development of teachers. This qualitative piece of research adopted a case study design. The participants were four Early Childhood Education teachers from the province of A Coruña (Spain). Data were collected through in-depth interviews. The information was processed through content analysis strategies. The re-
\end{abstract}

1 Dirección para correspondencia (correspondence address):

Alba Souto-Seijo. Universidade da Coruña, Facultad de Ciencias de la Educación, Campus de Elviña, s/n, 15071, A Coruña (España). 
Oportunidades de aprendizaje y formación docente: una mirada desde las Ecologías de Aprendizaje

Alba Souto-Seijo, Iris Estévez y Olalla Sande

de Educación Infantil de la provincia de A Coruña. La técnica de recogida de datos empleada ha sido la entrevista en profundidad. La información fue procesada mediante estrategias de análisis de contenido. Los resultados muestran que los docentes llevan a cabo múltiples actividades formativas para mantenerse actualizados, entre las que destacan los cursos que oferta la propia Administración educativa, a través de los Centros de Formación y Recursos, y las reuniones con otros profesionales. Las Ecologías de Aprendizaje se presentan como un marco útil desde el cual poder integrar y optimizar las diversas experiencias de aprendizaje, para entender de forma holística los diversos elementos que determinan el proceso de desarrollo profesional de los docentes del caso de estudio y el potencial de sus sinergias.

\section{Palabras clave:}

Desarrollo profesional docente; ecologías de aprendizaje; educación infantil; aprendizaje a lo largo de la vida; aprendizaje formal; aprendizaje no formal; aprendizaje informal; aprendizaje autodidacta. sults show that teachers carry out multiple training activities to keep up-to-date including courses offered by the educational administration, through the Training and Resource Centres, and meetings with other professionals. Learning Ecologies are presented as a useful framework from which it is possible to integrate and optimize various learning experiences, understanding holistically the manyfold elements that determine the professional development of the four teachers in the case study and the potential of their synergies.

\section{Key words:}

Teacher professional development; learning ecologies; Early Childhood Education; life-long learning; formal learning; non-formal learning; informal learning; self-training.

\section{Résumé:}

L'une des caractéristiques les plus pertinentes de la société de l'information et de la connaissance est que l'apprentissage ne se déroule plus seulement dans les établissements d'enseignement formels, mais aussi dans des espaces non formels et informels. Les technologies numériques nous donnent la possibilité d'apprendre à tout moment et en tout lieu, en brisant les barrières spatio-temporelles, de sorte que, de nos jours, les expériences d'apprentissage sont illimitées. Ainsi, cet article vise à analyser comment différentes opportunités d'apprentissage et de formation contribuent au développement professionnel des enseignants. Cette recherche, de nature qualitative, a été développée selon la tradition de la recherche par étude de cas. Les participants qui composent le cas sont quatre enseignants de l'éducation de la petite enfance dans la province de La Corogne. La technique de collecte de données utilisée était l'entretien approfondi. Les informations ont été traitées au moyen de stratégies d'analyse de contenu. Les résultats montrent que les enseignants réalisent de multiples activités de formation pour se maintenir à jour, parmi lesquelles se distinguent les cours offerts par l'administration de l'éducation elle-même, à travers les centres de formation et de ressources, et les rencontres avec d'autres professionnels. Les écologies d'apprentissage sont présentées comme un cadre utile à partir duquel intégrer et optimiser les diverses expériences d'apprentissage, afin de comprendre de manière holistique les différents éléments qui déterminent le processus de développement professionnel des enseignants de l'étude de cas et le potentiel de leurs synergies.

\section{Mots clés:}

Développement professionnel des enseignants; écologies dıapprentissage; éducation de la petite enfance; apprentissage tout au long de la vie; apprentissage formel; apprentissage non formel; apprentissage informel; apprentissage autodirigé. 
Fecha de recepción: 13-01-2021

Fecha de aceptación: 02-03-2021

\section{Introducción}

Se presume incuestionable que Internet y las tecnologías digitales han jugado un papel trascendental en la conformación y evolución de la Sociedad de la Información y de la Sociedad del Conocimiento, dado que han impactado poderosamente en todos los ámbitos de la actividad humana, transformando nuestros hábitos, la manera en que interactuamos, nos comunicamos y, por consiguiente, también la forma en que aprendemos. Las tecnologías han incrementado la velocidad con la que se produce, distribuye y renueva el conocimiento (Duart y Mengual-Andrés, 2014; González-Sanmamed, Sangrà, Souto-Seijo, y Estévez, 2020), lo que exige a los ciudadanos en general, y al profesorado en particular, entender la formación y el aprendizaje como procesos continuos que se desarrollarán a lo largo y ancho de la vida. Así pues, es un hecho ineludible que cada vez son mayores los desafíos a los que se enfrentan los docentes, pues su tarea profesional consiste en promocionar eficazmente el proceso de enseñanza-aprendizaje en escenarios cada vez más plurales, versátiles, y en constante cambio (Estévez et al., 2020; SoutoSeijo, Estévez, Romero et al., 2020).

Estos nuevos, amplificados y heterogéneos contextos proporcionan a los individuos múltiples formas de involucrarse y aprovechar las oportunidades de aprendizaje y desarrollo profesional que se les presentan. Desde una perspectiva ecológica, estas oportunidades se pueden identificar con el uso de diferentes recursos (con base tecnológica o no) en el proceso de aprendizaje o formación de un docente; también con el establecimiento de relaciones o interacciones con otros individuos (del ámbito personal o profesional) que constituyan una mejora en el desempeño del profesorado; y, por último, con la realización de diferentes actividades o acciones de aprendizaje que contribuyan al proceso de aprendizaje y actualización del docente (González-Sanmamed, MuñozCarril y Santos-Caamaño, 2019).

Como adelantábamos en líneas previas, en esta nueva realidad en la que han incrementado las posibilidades de aprendizaje y formación, emerge el concepto de Ecologías de Aprendizaje (EdA), definido como 
Oportunidades de aprendizaje y formación docente: una mirada desde las Ecologías de Aprendizaje

Alba Souto-Seijo, Iris Estévez y Olalla Sande

el entramado de contextos y elementos, de diversa naturaleza que las personas utilizan y gestionan para formarse y aprender (Barron, 2006; Jackson, 2013; Romeu-Fontanillas, Guitert-Catasús, Raffaghelli y Sangrà, 2020). De acuerdo con González-Sanmamed, Souto-Seijo, González et al. (2019) estos elementos pueden hacer referencia a la asistencia a cursos presenciales institucionalizados, pero también a la participación en redes sociales o incluso al visionado de algún programa de televisión. Así, este constructo nos proporciona un marco útil y holístico para analizar el desarrollo profesional docente en la actualidad, pues permite reconocer e integrar todas las formas en que el aprendizaje puede ocurrir hoy en día (Estévez et al., 2020; Looi, 2001; Romeu-Fontanillas et al., 2020; Valdés, Pilz, Rivero, Machado, Walder, 2013).

En el ámbito de las EdA se entiende al aprendiz como creador y actor principal de sus experiencias de aprendizaje. Experiencias que incorporan la educación formal y no formal, el aprendizaje informal y la autoformación. En este marco, Peña (2013) hace una interesante propuesta para definir los diferentes escenarios de aprendizaje, tomando como criterio de análisis dos ejes: enseñanza estructurada/sin estructurar y aprendizaje planificado/sin planificar. Consecuentemente, se identifican las cuatro modalidades de aprendizaje que definiremos y analizaremos a continuación.

En primer lugar, se dispone el Aprendizaje formal. Este tiene lugar en instituciones específicamente dedicadas a la enseñanza. La formación del aprendiz está estructurada, tanto en lo que respecta al contenido como al formato. Además, el proceso de aprendizaje generado en este escenario se caracteriza por ser intencional, por tener una duración determinada, y por establecer unos objetivos concretos que, una vez alcanzados, suponen la obtención de un certificado. Un ejemplo de aprendizaje formal es el que se adquiere en las Escuelas Oficiales de Idiomas, en los Másteres o Grados Universitarios, o en los cursos de formación ofertados por instituciones públicas como los Centros de Formación y Recursos (CFR). Es preciso destacar en este punto que, en la comunidad autónoma de Galicia -contexto en el que se ha llevado a cabo el presente estudio- la formación permanente del profesorado se rige por el Decreto 74/2011, del 14 de abril. De acuerdo con este Decreto, es la Administración educativa quien planifica las actividades de formación permanente, garantizando una oferta diversificada y gratuita de las mismas. De este modo, es el Centro Autonómico de Formación e Innova- 
ción (CAFI), quien se encarga de diseñar y elaborar los planes anuales de formación, y los Centros de Formación y Recursos (CFR), quienes ejecutan todas aquellas acciones formativas previstas en dichos planes.

En segundo lugar, se halla el Aprendizaje No Formal, que también puede suceder en un entorno de enseñanza formal (por ejemplo, un congreso). Sin embargo, la planificación del itinerario de aprendizaje es mucho más flexible que en la enseñanza formal, por lo que se suele adaptar mejor a las peculiaridades y necesidades de cada persona (Souto-Seijo, Estévez, Iglesias et al., 2020). Asimismo, el aprendiz no suele haber planificado tan intensamente dicho itinerario, al no haberlo ligado a unos objetivos de aprendizaje explícitos y determinados a medio o largo plazo. Al igual que en los procesos de aprendizaje en contextos formales, la modalidad no formal puede conllevar la obtención de un certificado, pero no siempre sucede. Algunos ejemplos de actividades no formales de aprendizaje en el ámbito docente podrían ser: la asistencia a alguna charla o taller sobre un tema de actualidad, la implicación en congresos o jornadas, o la participación en MOOCs (Cursos abiertos masivos en línea).

En tercer lugar, el Aprendizaje Informal es aquel que carece de toda estructura formal (tanto en lo que respecta a la ausencia de instituciones educativas respaldando el proceso, como en la carencia de un diseño explícito del itinerario de aprendizaje). Esto supone que no tiene una duración específica, ni una planificación particular, ni unos objetivos concretos, por lo que no implica la obtención de una certificación (CEDEFOP, 2014). En muchas ocasiones, tiene lugar de forma casual a partir de las experiencias aleatorias de la cotidianidad $y$, habitualmente, de forma inconsciente y fortuita. De hecho, en algunas ocasiones, podría identificarse con el denominado "aprendizaje invisible", metáfora generada por Cobo y Moravec (2011) para describir los aprendizajes de carácter experiencial, inconsciente y no sistematizado que, en la mayoría de los casos, puede no ser reconocible, ni exportable. Una de las mejores representaciones del aprendizaje informal es el que deriva de la interacción con otras personas en el ámbito profesional o personal, como es el caso de las charlas con compañeros de trabajo, o algo tan usual como leer el periódico.

Por último, definimos y caracterizamos el Aprendizaje Autodidacta, en ocasiones, también denominado autoformación. Como el aprendizaje informal, el aprendizaje autodidacta se puede dar en cualquier mo- 
Oportunidades de aprendizaje y formación docente: una mirada desde las Ecologías de Aprendizaje

Alba Souto-Seijo, Iris Estévez y Olalla Sande

mento y lugar, y no se cierne a las barreras de las instituciones formales de formación. Sin embargo, sí obedece a una planificación de objetivos de aprendizaje, así como a la consecución de metas establecidas por el propio aprendiz. En el aprendizaje autodidacta existe una total consciencia y volición de aprender, a diferencia del aprendizaje informal (Estévez et al., 2020; Peña, 2013). Este aprendizaje deriva de la formación en la que los sujetos participan de forma independiente (Marcelo y VaiIlant, 2018), por lo que representa una respuesta más natural y ajustada a la hora de afrontar los continuos retos que plantea la sociedad actual (Guglielmino, 2008). Un ejemplo de aprendizaje autodidacta se podría vincular al desarrollo de un blog educativo, a través de la búsqueda de información en diferentes canales digitales, o mediante la consulta de manuales específicos en respuesta a una inquietud particular.

Después de definir las diferentes modalidades de aprendizaje profesional docente, se presume necesario señalar que, desde un prisma ecológico de la realidad del profesorado, estos escenarios de desarroIlo profesional, lejos de ser excluyentes, deben ser entendidos como complementarios. Así, dependiendo de la naturaleza del conocimiento, competencia o habilidad que el docente desee aprender, podrá optar por la modalidad que considere más adecuada. De esta manera, las limitaciones que, por ejemplo, puede presentar la modalidad de formación formal por no ceñirse estrictamente a las necesidades individuales y particulares de cada docente (Muñoz-Carril, González-Sanmamed, y Hernández-Sellés, 2013), pueden verse subsanadas si se complementa con ciertas actividades de formación no formal, autodidacta o alguna acción de aprendizaje informal. En este sentido, tal y como defienden diferentes autores, parece que se ha producido un incremento del protagonismo del aprendizaje informal y autodidacta en los procesos de desarrollo profesional y de formación permanente de los profesionales de la enseñanza (p.e. Marcelo, 2010; Marcelo y Vaillant, 2018).

De este modo, como relatábamos en líneas previas, el paradigma ecológico se basa en un constructo todavía en estado embrionario y, consecuentemente, poco estudiado en el ámbito del aprendizaje (Han y Ellis, 2020). A pesar de ello, las EdA se presentan como un marco de análisis eficaz para el estudio de procesos complejos y multidimensionales, como es el caso del desarrollo profesional docente (p.e. GonzálezSanmamed et al., 2020; Sangrá, Raffaghelli, y Guitert, 2019). Además, tal y como se expuso con anterioridad, las dinámicas formativas de los 
docentes se han transformado en los últimos años, dando lugar a itinerarios de aprendizaje en los que se combinan diversos escenarios y contextos. Esto nos ofrece un panorama susceptible de ser analizado, para poder diseñar y crear (desde una perspectiva individual e institucional) planes de formación más ajustados a las necesidades e intereses de cada docente, y que den respuesta a las demandas del sistema educativo actual. Por todo ello, el presente trabajo se ha llevado a cabo con el objetivo de determinar cómo y en qué medida las diferentes modalidades de aprendizaje contribuyen al desarrollo profesional del profesorado de nuestro caso de estudio, que imparte docencia en la etapa de Educación Infantil. Las preguntas de investigación que han guiado el estudio han sido las siguientes: a) ¿Qué acciones Ilevan a cabo los docentes de Educación Infantil de nuestro caso de estudio para mantenerse actualizados?, y b) ¿En qué medida son valoradas esas diferentes modalidades de aprendizaje por los docentes de nuestro caso?

\section{Marco empírico}

Esta investigación, de corte cualitativo-interpretativo, se ha desarrollado a través de la tradición de investigación del Estudio de Caso. Esta es una de las corrientes más comúnmente empleada en el campo de la investigación educativa en general y, particularmente, en el estudio de las Ecologías de Aprendizaje (véase, por ejemplo, Barron, 2006; Barron, Martin, y Roberts, 2007; Martínez-Rodríguez y Benítez-Corona, 2020; Romeu-Fontanillas, Guitert, Raffaghelli, y Sangrà, 2020). Concretamente, este estudio responde a un diseño holístico de múltiples casos (Yin, 2018). Por un lado, es holístico, ya que solo se ha utilizado una unidad de análisis y, por otro, es múltiple, debido a que el estudio lo conforman cuatro casos.

\section{Participantes}

Mediante un muestreo de casos homogéneos (Patton, 2002) se ha seleccionado a los cuatro docentes de Educación Infantil, tres mujeres y un hombre, que conforman el estudio. Cabe mencionar que todos ellos imparten docencia en diferentes centros educativos de la provincia de A Coruña (España) y que tienen edades comprendidas entre 34 y 48 
Oportunidades de aprendizaje y formación docente: una mirada desde las Ecologías de Aprendizaje

Alba Souto-Seijo, Iris Estévez y Olalla Sande

años. A continuación, se exponen los criterios de elegibilidad de los participantes que se han establecido previamente: a) búsqueda activa de formación, b) empleo de metodologías innovadoras, y c) integración de las tecnologías digitales en el aula.

\section{Técnica de recogida de datos}

La técnica de recogida de datos empleada con el objetivo de identificar las actividades que contribuyen al desarrollo profesional de los docentes participantes en el Estudio de Caso ha sido la entrevista en profundidad (Creswell y Creswell, 2018). Concretamente, se caracteriza por ser una entrevista semidirigida o semiestructurada (Verd y Lozares, 2016), ya que se ha diseñado un guion previo con diferentes preguntas con la finalidad de asegurarse de que se abordaban los temas claves con los cuatro participantes. Es preciso mencionar que para la elaboración del guion se tuvo en consideración la literatura existente en torno al tópico de desarrollo profesional docente y al de Ecologías de Aprendizaje. Una vez diseñado el guion, éste fue sometido a un juicio de tres expertos, cuyas sugerencias fueron tomadas en cuenta para elaborar la estructura definitiva. Esta, finalmente, constó de una batería de 56 preguntas agrupadas en los siguientes bloques: formación y actualización, colaboración entre instituciones, transferencia y TIC. Por último, cabe señalar que la entrevista se llevó a cabo presencialmente y de manera individual con cada docente. La duración aproximada de cada encuentro fue de entre 45-90 minutos.

\section{Procedimiento}

Primeramente, se contactó con los docentes que cumplían los criterios descritos anteriormente para invitarlos a participar en el estudio. En este primer contacto, se les informó sobre el propósito y duración de la investigación, y se garantizó la protección de su identidad, mediante el uso de nombres ficticios, y la confidencialidad de la información, dado que los datos recabados solo serán accesibles de cara a la investigación y, en ningún caso, se hará un uso inapropiado de los mismos. Asimismo, se acordó con cada uno de ellos el lugar, día y hora de la entrevista. Los encuentros fueron grabados, previa autorización de los participantes, para registrar fielmente su testimonio. Posteriormente, las cuatro entrevistas 
fueron transcritas literalmente y analizadas. Para la identificación de las transcripciones se han empleado los siguientes códigos: E1, E2, E3, E4; asignando un número a cada persona entrevistada (ver Tabla 1).

Tabla 1

Códigos empleados para la identificación de las entrevistas

\begin{tabular}{ccccc}
\hline & Ana & Nicolás & Sara & Zeltia \\
\hline Código & E1 & E2 & E3 & E4 \\
\hline
\end{tabular}

\section{Análisis de datos}

El análisis de los datos se desarrolló de acuerdo con el procedimiento establecido por Miles, Huberman, y Saldaña (2014). Durante la primera fase, condensación de los datos, tuvo lugar la decodificación-codificación de la información. Es preciso señalar que se seleccionó la información que estaba vinculada a las preguntas de investigación, recurriendo así al denominado análisis de contenido (Bengtsson, 2016). En el presente estudio se combinaron estrategias de codificación inductivas y deductivas, por lo que se llevó a cabo una codificación mixta (Saldaña, 2016). En la Figura 1 se presenta el esquema final de categorías obtenido después de alcanzar la saturación teórica.

A continuación, durante la fase de disposición de los datos, se crearon matrices que sirvieron de apoyo para crear, en la fase de obtención y verificación de conclusiones, los informes de investigación y elaborar las conclusiones que aquí se presentan. Todo este proceso de análisis se realizó con la ayuda del programa de análisis cualitativo denominado Atlas.ti (versión 7).

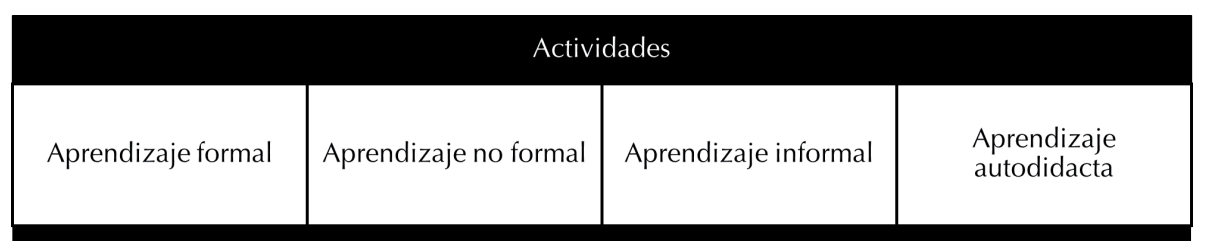

Figura 1. Sistema de categorías. 
Oportunidades de aprendizaje y formación docente: una mirada desde las Ecologías de Aprendizaje

Alba Souto-Seijo, Iris Estévez y Olalla Sande

\section{Resultados y Discusión}

Seguidamente, se describen aquellos aspectos que han emergido del análisis de los cuatro casos y que permiten determinar cómo las diferentes modalidades de aprendizaje contribuyen al desarrollo profesional del profesorado de Educación Infantil participante en este estudio y, por tanto, al enriquecimiento de su Ecología de Aprendizaje.

\section{Aprendizaje formal}

En primer lugar, cabe señalar que los cuatro docentes participan, sobre todo, en acciones formativas que provienen de la propia administración educativa. Este hallazgo apoya la investigación previa de Fernández y Montero (2007), desarrollada en la Comunidad Autónoma de Galicia. Una posible hipótesis explicativa de este resultado puede venir determinada por: a) la gratuidad de las actividades ofertadas; b) la obtención de un certificado, lo que constituye un requisito esencial para el reconocimiento y la acreditación de la actividad formativa a efectos de promoción, concurso de traslados, etc.; y c) cuestiones de compatibilidad horaria, ya que estas actividades no son coincidentes con el horario lectivo del profesorado en el centro educativo. Todos estos aspectos están recogidos en la normativa que regula la formación permanente del profesorado en Galicia (el Decreto 74/2011, del 14 de abril y la Orden de 14 de mayo de 2013). Así, destacan los cursos de los Centros de Formación y Recursos (CFR), una institución dependiente de la Consellería de Cultura, Educación e Ordenación Universitaria, a los que los docentes recurren habitualmente dado que la oferta es amplia y les permite formarse en temáticas muy diversas.

Cabe señalar que, aunque el profesorado participante en este estudio de caso afirma realizar cursos tanto en línea como presenciales, todos ellos parecen estar de acuerdo en que prefieren la formación presencial. Este resultado coincide con lo manifestado por García-Ruiz y Castro (2012), quienes afirman que la modalidad presencial continúa siendo la preferida por los docentes de Educación Infantil y Primaria. De esta, destacan el trato personal y la posibilidad de resolver las dudas de manera más rápida y eficaz. No obstante, también reconocen que la formación en línea tiene importantes beneficios, ya que les permite formarse en cualquier momento y lugar. Tal y como explica Nicolás, "en los cursos 
en línea si tú tienes una duda, puedes escribir un correo electrónico y te lo contestan al día siguiente... pero el proceso es un poco más lento... Aunque lo valoro positivamente por el hecho de que tú distribuyes tu tiempo, no tienes un horario cerrado ni que desplazarte" (E2, p. 3).

Otra de las actividades formativas institucionales más valoradas son los Proyectos de Formación en Centros, dependientes de la misma Consellería, y que permiten a los centros educativos diseñar sus propios planes de formación en función de las necesidades detectadas. De acuerdo con autores como Fernández y Montero (2007) y García-Ruiz y Castro (2012) este modelo de formación más contextualizada es lo que hace que estas propuestas sean altamente valoradas por este colectivo. Según el profesorado participante en el Estudio de Caso los Proyectos de Formación en Centros no solo contribuyen a su desarrollo profesional, sino que mejoran la dinámica del centro porque en ellos colaboran docentes de la propia escuela. Ana comenta que este tipo de formación "ayuda a que vayas trayendo a tu centro lo que tú antes igual tenías dificultades para buscar en otros sitios" (E1, p. 1).

De igual forma, esta misma docente también manifiesta que realiza cursos del Instituto Nacional de Tecnologías Educativas y de Formación del Profesorado (INTEF), la unidad del Ministerio de Educación, Cultura y Deporte responsable de la integración de las tecnologías digitales en los centros educativos de las etapas no universitarias.

Finalmente, es preciso mencionar que una de las docentes, Sara, está cursando Antropología, dado que siente la necesidad de conocer más al ser humano y así comprender mejor a su alumnado. Poniendo de manifiesto, nuevamente, la positiva opinión referida a que la formación formal le merece a los participantes en nuestros casos de estudio.

\section{Aprendizaje no formal}

Los continuos cambios que acontecen en la sociedad actual impulsan a los docentes a llevar a cabo otro tipo de acciones de formación complementarias que les permitan ser competentes y dar respuesta a las necesidades e intereses del alumnado. En lo que concierne al aprendizaje no formal cabe destacar que tres de los docentes (Zeltia, Nicolás y Ana), forman parte de grupos de trabajo que se reúnen mensualmente, ya que esta actividad les permite seguir formándose y desarrollándose profesionalmente en temas concretos en los que están interesados. Este hallazgo 
Oportunidades de aprendizaje y formación docente: una mirada desde las Ecologías de Aprendizaje

Alba Souto-Seijo, Iris Estévez y Olalla Sande

confirma lo ya encontrado por Barquín (2014) y Pérez-Granados (2018) en dos estudios realizados en Andalucía, y en los que se determina que los grupos de trabajo son una de las modalidades más elegidas por los docentes para formarse. De acuerdo con Aneas-Novo, Sánchez-Rodríguez y Sánchez-Rivas (2019), y Krichesky y Murillo (2018), detrás del éxito de este tipo de acciones formativas podría estar la cohesión grupal generada a partir del trabajo colaborativo que se lleva a cabo.

La cuarta docente, Sara, si bien no participa en este tipo de acciones, sí ha realizado diversas estancias en otros centros educativos, tanto a nivel nacional como internacional. Esta profesora considera que es una experiencia muy enriquecedora, que no solo le proporciona aprendizajes que propulsan su desarrollo profesional, sino también su desarrollo personal: "es algo muy enriquecedor cuando haces visitas y te dan la oportunidad de formar parte de otra manera de hacer... Es muy positivo a todos los niveles: para el aprendizaje y para la vida" (E3, p. 3). A pesar de que los otros tres docentes no han tenido la oportunidad de realizar acciones formativas relativas a la realización de estancias en otros contextos educativos (nacionales o internacionales), todos coinciden en que les gustaría embarcarse en este tipo de experiencias para ampliar su formación. En este sentido, como bien indican Vázquez-Rodríguez, García-Álvarez y Santos-Rego (2021), este tipo de acciones vinculadas a la movilidad, contribuyen, en gran medida, a la adquisición de competencias y el establecimiento de redes personales de aprendizaje.

Por último, cabe mencionar que los docentes de nuestro Estudio de Caso también asisten a congresos, seminarios, jornadas y encuentros. Este tipo de actividades les resultan muy interesantes, ya que suelen ser más prácticas y dinámicas que los cursos a los que normalmente acuden, que suelen caracterizarse por ser más teóricos. Asimismo, en ocasiones participan en acciones formativas organizadas por fundaciones, como es el caso de la Fundación Barrié, aunque admiten que acudir a este tipo de actividades les resulta complicado debido a que los horarios en los que normalmente se celebran no son compatibles con su tarea docente.

\section{Aprendizaje informal}

En tercer lugar, en relación con el aprendizaje informal, es conveniente destacar que todos los docentes participantes declaran que los encuentros informales con compañeros, esos que se producen de manera causal 
y cotidiana, les aportan tanto como las formaciones institucionalizadas, ya que en ellos intercambian información y experiencias, lo que entienden de gran utilidad para mejorar su práctica diaria. Estos resultados coinciden con lo manifestado por Marcelo y Vaillant (2018), quienes afirman que las interacciones entre pares juegan un papel fundamental en la adquisición de nuevos conocimientos y capacidades.

De igual modo, reconocen que acuden a ellos para solventar las dudas que les van surgiendo en el día a día de la escuela, y que en diversas ocasiones son los propios compañeros los que los motivan para continuar aprendiendo. Aunque mayoritariamente hacen referencia a los docentes de su propio centro o de otros centros cercanos, cabe señalar que también contactan con otros profesionales considerados expertos en determinadas temáticas a través de diversos recursos tecnológicos como pueden ser el correo electrónico, los blogs o las redes sociales.

Asimismo, también hacen referencia a la oportunidad de formación que les supone impartir conferencias, tener estudiantes en prácticas en sus aulas, realizar viajes, visionar películas, etc. Finalmente, Sara destaca la importancia de su familia, ya que todos son muy inquietos, comparten sus vivencias y aprenden los unos de los otros: "En mi casa somos cuatro hermanos y cada uno estudió una cosa, pero somos todos tan de compartir y vivimos todo tan intensamente, que cuando alguno está metido en un proyecto, nos empapa, formamos parte... Todos aprendemos de todos" (E3, p. 7).

\section{Aprendizaje autodidacta}

En cuarto lugar, los resultados ponen de manifiesto que los cuatro docentes participantes en el Estudio de Caso consideran fundamental el aprendizaje autodidacta, ya que, como establecen Marcelo y Vaillant (2010), les ayuda a ampliar y complementar la información recibida a través de otros canales (cursos, jornadas, encuentros con compañeros, etc.) según sus necesidades e intereses particulares. En palabras de Zeltia: "A partir de los conocimientos que adquiero en diferentes cursos o aspectos que surgen hablando con otros docentes, intento buscar información en internet para ampliar un poco o comprarme libros relacionados con el tema (...) o, por ejemplo, alguna cosa que escuchas más de pasada y buscas en internet o en libros en qué consiste, en qué se basa... por si puede ser interesante o tener aplicación en el aula" (E4, p. 3). 
De este modo, se observa como en la autoformación juegan un gran papel las tecnologías digitales, pues les permiten acceder de manera rápida y sencilla al conocimiento y gestionar un proceso de aprendizaje autónomo (Marcelo y Vaillant, 2018). Las actividades más nombradas por estos docentes son las siguientes: búsqueda en Internet, consulta de blogs educativos de otros docentes que comparten sus vivencias, lectura de libros o artículos de revistas, consulta de redes sociales para estar al tanto de información novedosa, etc. Este hallazgo está en línea con lo aportado por González-Sanmamed, Souto-Seijo, González y Estévez (2019) en una investigación llevada a cabo con profesorado de la misma etapa educativa.

Por último, los cuatro docentes hacen especial énfasis en la reflexión sobre la propia práctica educativa, aspecto que conviene resaltar, pues el análisis de la experiencia posibilita una mayor consciencia sobre las propias carencias en el conocimiento profesional (Marcelo, 2010). Concretamente, dos de ellos afirman que cumplimentan diariamente un registro de aula, lo que les ayuda analizar los puntos fuertes y débiles de sus intervenciones educativas para posteriormente diseñar acciones de mejora. En el caso de una de estas docentes cabe mencionar que todo el profesorado de su centro educativo comparte sus reflexiones con el resto trimestralmente, lo que genera muchos y valiosos aprendizajes.

Finalmente, uno de los resultados más significativos emanados de esta investigación se fundamenta en el hecho de que los cuatro docentes participantes en el Estudio de Caso están muy implicados en su formación, a la que dedican un gran número de horas y mucho esfuerzo. Además, todos ellos afirman que el aprendizaje que van adquiriendo a través de las diferentes modalidades lo transfieren al aula con el objetivo de mejorar el proceso de enseñanza-aprendizaje. Este resultado es esperanzador, ya que como apunta Imbernón (2007), los aprendizajes derivados de las actividades formativas no siempre se llevan a la práctica y ello limita las posibilidades de mejora continua que se esperan de la formación y el desarrollo profesional continuo.

\section{Conclusiones}

En este último punto se exponen las conclusiones extraídas en este estudio. En primer lugar, es conveniente señalar que los resultados ponen 
de manifiesto que los docentes de Educación Infantil participantes en el Estudio de Caso se involucran y aprovechan las diversas oportunidades de aprendizaje que se les presentan en su día a día. De este modo, se puede afirmar que no solo realizan acciones formativas formales, como los cursos ofertados por los Centros de Formación y Recursos (CFR) o los Proyectos de Formación en Centros, sino que también llevan a cabo otro tipo de actividades y acciones ubicadas en escenarios no formales (p.e. participación en grupos de trabajo) o informales (p.e. encuentros informales con compañeros). Asimismo, y en gran medida gracias a los recursos tecnológicos, también se forman de manera autodidacta (p.e. consulta de blogs educativos).

En este contexto que acabamos de describir, en el que el aprendizaje docente no está constreñido a un espacio o momento temporal determinado, tiene especial relevancia la perspectiva ecológica, dado que permite entender la integración y las sinergias entre las diferentes modalidades formativas $y$, en consecuencia, promueve que cada persona genere su propio itinerario de aprendizaje atendiendo a la diversidad de oportunidades disponibles. De hecho, como ya apuntaban Colley, Hodkinson y Malcolm (2002) o Smith (2008) existe un creciente desdibujado entre los ejes que conforman los límites entre los escenarios de aprendizaje descritos. Así, los resultados de este trabajo avalan una perspectiva en la cual todas las modalidades de formación cumplen un propósito diferencial dependiendo del contexto y no son excluyentes, sino complementarias. La combinación de las diferentes tipologías de actividades, minimizan las limitaciones que presentan cada una de ellas.

Los resultados de este estudio exhortan hacia la necesidad de generación de espacios que supongan el aprovechamiento de las diversas oportunidades de aprendizaje que el contexto actual ofrece a los docentes de Educación Infantil. En este sentido, tal y como determina la Comisión Europea (2018), se considera prioritario poner en marcha diversas acciones que permitan valorar todos los aprendizajes adquiridos por el profesorado, independientemente del contexto donde se generen, pues esta declaración revertirá directamente en una mejora del desarroIlo profesional de los docentes y, por tanto, en la calidad del proceso de enseñanza-aprendizaje (Souto-Seijo, Estévez, Iglesias et al., 2020). No obstante, esta valoración no se circunscribe a una mera acreditación de la formación realizada, -con lo que, ciertamente, se podría estar contraviniendo la idiosincrasia de los aprendizajes informales (Sangrá y Whe- 
Oportunidades de aprendizaje y formación docente: una mirada desde las Ecologías de Aprendizaje

Alba Souto-Seijo, Iris Estévez y Olalla Sande

eler, 2013)- sino que se debería vincular con la cesión de herramientas, tiempo y espacios que fomenten la generación de escenarios de aprendizaje que no sean exclusivamente formales.

Finalmente, se considera oportuno citar las principales limitaciones de este estudio. En primer lugar, cabe mencionar que la información fue recabada exclusivamente a través de entrevistas. Es por ello que, de cara a próximas investigaciones, se tratará de complementar los datos de las entrevistas con la información obtenida mediante otras técnicas como la observación o el focus group. Asimismo, teniendo en cuenta los resultados obtenidos, en futuros estudios se contempla el análisis de las interrelaciones generadas entre los diversos elementos de las Ecologías de Aprendizaje, procurando profundizar en las influencias y sinergias que se producen entre las acciones o actividades de aprendizaje y las interacciones, los recursos empleados, o los mecanismos motivacionales que los docentes ponen en marcha cuando participan en los diversos escenarios de aprendizaje (formal, no formal, informal o autodidacta) para seguir formándose a lo largo de su trayectoria vital.

\section{Referencias}

Aneas-Novo, C., Sánchez-Rodríguez, J., y Sánchez-Rivas, E. (2019). Valoración de la formación del profesorado: comparativa entre autoformación y formación presencial. Nueva época, 21, 94-108. doi: 10.24965/gapp.v0i21.1056

Barquín, J. (2014). Informe sobre el profesorado de Andalucía y la formación permanente. Junta de Andalucía.

Barron, B. (2006). Interest and self-sustained learning as catalysts of development: A learning ecology perspective. Human Development, 49(4), 193-224. doi: $10.1159 / 000094368$

Barron, B., Martin, C. K., y Roberts, E. (2007). Sparking self-sustained learning: report on a design experiment to build technological fluency and bridge divides. International Journal of Technology and Design Education, 17(1), 75-105. doi: 10.1007/s10798006-9002-4

Bengtsson, M. (2016). How to plan and perform a qualitative study using content analysis. NursingPlus Open, 2, 8-14. doi: 10.1016/J.NPLS.2016.01.001

CEDEFOP (2014). Terminology of European education and training policy. A selection of 130 key terms. Office for Official Publications of the European Communities. https:// bit.ly/2Tmxv2m

Cobo, C., y Moravec, J. W. (2011). Aprendizaje Invisible. Hacia una nueva ecología de la educación. Barcelona: Publicacions i Edicions de la Universitat de Barcelona.

Colley, H., Hodkinson, P., y Malcolm, J. (diciembre, 2002). Non-formal learning: map- 
Oportunidades de aprendizaje y formación docente: una mirada desde las Ecologías de Aprendizaje

Alba Souto-Seijo, Iris Estévez y Olalla Sande

ping the conceptual terrain. Learning and Skills Research Network Annual Conference, University of Warwick. Recuperado de https://cutt.ly/RrsXGMR

Comisión Europea (2018). Recomendación del Consejo, de 22 de mayo de 2018, relativa a las competencias clave para el aprendizaje permanente. Recuperado de https://bit. ly/2zT9Gbz

Creswell, J. W., y Creswell, J. D. (2018). Research Design: Qualitative, Quantitative \& Mixed Methods Approaches. London: Sage Publications.

Decreto 74/2011, del 14 de abril, por el que se regula la formación permanente del profesorado que imparte enseñanzas establecidas en la LOE, en centros educativos sostenidos con fondos públicos de la Comunidad Autónoma de Galicia. Boletín Oficial del Estado, 88 (6 de mayo de 2011), 7921-7938.

Duart, J. M., y Mengual-Andrés, S. (2014). Impacto de la Sociedad del Conocimiento en la universidad y en la comunicación científica. RELIEVE. Revista Electrónica de Investigación y Evaluación Educativa, 20(2), 1-12. doi: 10.7203/relieve.20.2.4343.

Estévez, I., Souto-Seijo, A., Sande, O., y González-Sanmamed, M. (2020). Aprendizaje Profesional Docente a través de la Autoformación: un Análisis Cualitativo de las Ecologías de Aprendizaje. En S. Oliveira e Sá, F. Freitas, P. Castro, M. González-Sanmamed, y A. P. Costa (Eds.), Investigación Cualitativa en Educación: avances y desafíos (Vol. 2, pp. 437-447). En https://doi.org/10.36367/ntqr.2.2020.437-447

Fernández, M. D., y Montero, M. L. (2007). Que pensan os asesores e os profesores sobre as modalidades de formación? Eduga: Revista Galega do Ensino, 49, 74-88. Recuperado de https://bit.ly/2VmeWy3.

García-Ruiz, R., y Castro, A. (2012). La formación permanente del profesorado basada en competencias: Estudio exploratorio de la percepción del profesorado de Educación Infantil y Primaria. Educatio Siglo XXI, 30(1), 297-322. Recuperado de https:// bit.ly/2ZmR6jP.

González Sanmamed, M., Muñoz-Carril, P. C., y Santos Caamaño, F. J. (2019). Key components of learning ecologies: A Delphi assessment. British Journal of Educational Technology, 5(4), 1639-1655. doi: 10.1111/bjet.12805

González-Sanmamed, M., Sangrà, A., Souto-Seijo, A., y Estévez, I. (2020). Learning ecologies in the digital era: challenges for higher education. Publicaciones, 50(1), 83-102. doi: 10.30827/publicaciones.v50i1.15671

González-Sanmamed, M., Souto-Seijo, A., González, I., y Estévez, I. (2019). Aprendizaje informal y desarrollo profesional: análisis de las ecologías de aprendizaje del profesorado de educación infantil. Edutec. Revista Electrónica De Tecnología Educativa, (68), 70-81. doi: 10.21556/edutec.2019.68.1305

Guglielmino, L. (2008). Why self-directed learning? International Journal of Self-Directed Learning, 5(1), 1-14.

Han, F., y Ellis, R. (2020). Personalised learning networks in the university blended learning context. Comunicar, 28(62), 19-30. doi:10.3916/C62-2020-02

Imbernón, F. (2007). Diez ideas clave. La formación permanente del profesorado. Nuevas ideas para formar en la innovación y el cambio. Barcelona: Graó.

Jackson, N. (2013). The Concept of Learning Ecologies. En N. Jackson y G. B. Cooper 
Oportunidades de aprendizaje y formación docente: una mirada desde las Ecologías de Aprendizaje

Alba Souto-Seijo, Iris Estévez y Olalla Sande

(Eds.), Lifewide Learning Education and Personal Development (pp. 1-21). Recuperado de https://bit.ly/2Bujbi1

Krichesky, G. J., y Murillo F. J. (2018). La colaboración docente como factor de aprendizaje y promotor de mejora. Un estudio de casos. Educación XX1, 21(1), 135-156, doi: 10.5944/educXX1.20181

Looi, C. K. (2001). Enhancing learning ecology on the Internet. Journal of Computer Assisted Learning, 17(1), 13-20. doi: 10.1111/j.1365-2729.2001.00155.x

Marcelo, C. (2010). Autoformación para el siglo XXI. En J. Gairín (Coord.) Nuevas estrategias formativas para las organizaciones (pp. 141-170). Madrid: Wolters Kluwer.

Marcelo, C., y Vaillant, D. (2010). Desarrollo profesional docente: ¿cómo se aprende a enseñar? Madrid: Narcea Ediciones.

Marcelo, C., y Vaillant, D. (2018). Hacia una formación disruptiva de docentes. 10 claves para el cambio. Madrid: Narcea Ediciones.

Martínez-Rodríguez, R. C., y Benítez-Corona, L. (2020). The ecology of resilience learning in ubiquitous environments to adverse situations. Comunicar, 28(62), 43-52. doi: 10.3916/C62-2020-04

Miles, M. B., Huberman, A. M., y Saldaña, J. (2014). Qualitative Data Analysis. A Methods Sourcebook (3rd ed.). London: Sage Publications.

Muñoz-Carril, P. C., González-Sanmamed, M., y Hernández-Sellés, N. (2013): Pedagogical Roles and Competencies of University Teachers Practicing in the E-learning Environment. The International Review of Research in Open and Distance Learning, 14(3), 462-487. doi: 10.19173/irrodl.v14i3.1477

Orden de 14 de mayo de 2013 por la que se regula la convocatoria, el reconocimiento, la certificación y el registro de las actividades de formación permanente del profesorado en Galicia. Boletín Oficial del Estado, 96 (22 de mayo de 2013), 17697 a 17732.

Patton, M. (2002). Qualitative research and evaluation methods (3rd ed.). London: Sage Publications.

Peña, I. (2013). El PLE de investigación-docencia: el aprendizaje como enseñanza. En L. Castañeda y J. Adell (Eds.), Entornos Personales de Aprendizaje: Claves para el ecosistema educativo en red (pp. 93-110). Alcoy: Marfil.

Pérez-Granados, L. (2018). El grupo de trabajo como estrategia de formación permanente del profesorado. Aula de Encuentro, 20(1), 4-25. doi: 10.17561/ae.v20i1.1.

Romeu-Fontanillas, T., Guitert, M., Raffaghelli, J. -E., y Sangrà, A. (2020). Ecologías de aprendizaje para usar las TIC inspirándose en docentes referentes. Comunicar, 28(62). doi: 10.3916/c62-2020-03

Saldaña, J. (2016). The Coding Manual for Qualitative Researchers (3rd ed.). London: Sage Publications.

Sangrá, A., Raffaghelli, J. E., \& Guitert, M. (2019). Learning ecologies through a lens: Ontological, methodological and applicative issues. A systematic review of the literature. British Journal of Educational Technology, 50(4), 1619-1638. doi: 10.1111/bjet.12795

Sangrà, A., y Wheeler, S. (2013). Nuevas formas de aprendizaje informales: ¿o estamos formalizando lo informal? Revista de Universidad y Sociedad del Conocimiento, 10, 107-115. doi: 10.7238/rusc.v10i1.1689 
Oportunidades de aprendizaje y formación docente: una mirada desde las Ecologías de

Smith, M. K. (2008). Informal learning: Theory, practice and experience. Recuperado de https://bit.ly/2K7zdD8

Souto-Seijo, A., Estévez, I., Iglesias, V., y González-Sanmamed, M. (2020). Entre lo formal y lo no formal: un análisis desde la formación permanente del profesorado. Educar 56(1), 97-107. doi: 10.5565/rev/educar.1095

Souto-Seijo, A., Estévez, I., Romero, P., y González-Sanmamed, M. (2020). Aprendizajes Formales, No Formales e Informales en la Era Digital: Contribuciones al Desarrollo Profesional Docente. En S. Oliveira e Sá, F. Freitas, P. Castro, M. González-Sanmamed, y A. P. Costa (Eds.), Investigación Cualitativa en Educación: avances y desafíos (Vol. 2, pp. 428-436). En https://doi.org/10.36367/ntqr.2.2020.428-436

Valdés, R., Pilz, D., Rivero, J., Machado, M. M., y Walder, G. (2013). Aportes conceptuales de la educación de personas jóvenes y adultas: hacia la construcción de sentidos comunes en la diversidad. Recuperado de https://bit.ly/2uRuzkS

Vázquez-Rodríguez, A., García-Álvarez, J., y Santos Rego, M. A. (2021). Movilidad internacional y empleabilidad: el impacto en el capital humano y social de la juventud. Educar, 57(1), 81-96. doi: 10.5565/rev/educar.1185

Verd, J. M., y Lozares, C. (2016). Introducción a la investigación cualitativa. Fases, métodos y técnicas. Madrid: Síntesis.

Yin, R. K. (2018). Case Study Research and Applications. Design and Methods (6th ed.). London: Sage Publication.

\section{Agradecimientos}

Este trabajo se ha elaborado en el marco del proyecto de investigación titulado: "Ecologías de aprendizaje en la era digital: nuevas oportunidades para la formación del profesorado de educación secundaria" (ECO4LEARN-SE), parcialmente financiado por el Ministerio de Ciencia, Innovación y Universidades (Referencia RTI2018-095690-B-I00). También gracias a la financiación recibida por una de las autoras de esta investigación, Iris Estévez, en el programa FPI del Ministerio de Economía y Finanzas (BES-2016-077330). 
